\title{
New electron energy transfer rates for vibrational excitation of $\mathbf{N}_{\mathbf{2}}$
}

\author{
A. V. Pavlov \\ Institute of Terrestrial Magnetism, Ionosphere and Radio-Wave Propagation, Russian Academy of Science (IZMIRAN), \\ Troitsk, Moscow Region, 142092, Russia, e-mail: avp@izmiran.troitsk.ru
}

Received: 12 February 1997 / Revised: 3 September 1997 / Accepted: 9 September 1997

\begin{abstract}
In this paper we present the results of a study of the electron cooling rate, the production rates of vibrationally excited $\mathrm{N}_{2}(v)$, and the production frequency of the $\mathrm{N}_{2}$ vibrational quanta arising from the collisions of electrons with unexcited $\mathrm{N}_{2}(0)$ and vibrationally excited $\mathrm{N}_{2}(1)$ molecules as functions of the electron temperature. The electron energy transfer rates for vibrational excitation of $\mathrm{N}_{2}$ have been calculated and fit to analytical expressions by use of the revised vibrationally excited $\mathrm{N}_{2}$ cross sections. These new analytical expressions are available to the researcher for quick reference and accurate computer modeling with a minimum of calculations.
\end{abstract}

Key words Atmospheric composition and structure · Thermosphere $\cdot$ Ionosphere $\cdot$ Modeling and forecasting

\section{Introduction}

The theoretical computation of electron temperature distribution in the ionosphere requires the knowledge of various heating, cooling, and energy flow processes. The energy transfer from electrons to neutral gases in the upper atmosphere and ionosphere is one of the dominant electron cooling processes in the ionosphere. The energy transfer rate from electrons to $\mathrm{N}_{2}$ have been calculated and fitted to analytical expressions by Stubbe and Varnum (1972) for vibrational excitation of $\mathrm{N}_{2}$. This cooling rate and the analytical expression are based on the $\mathrm{N}_{2}$ vibrational cross sections of Schulz (1964) and Engelhard et al. (1964). However, the measurements of Schulz (1976) and Haddad (1984) indicate much larger values for these cross sections. The cross sections of Schulz (1964) and those shown in Fig. 2.6 of Schulz (1976) differ by about a factor of 2. However, Schulz (1976), on the basis of some unpublished measurements by Wong et al., has suggested that even these larger cross sections should be doubled.

A normalization factor of 0.7 for Schulz's (1976) doubled nitrogen vibrational cross sections provides a good fit to a large body of experimental swarm data in nitrogen and argon-nitrogen mixture for the energy range above $1.7 \mathrm{eV}$ (Haddad, 1984). As a result, the first cross-section peak for the excitation of the nitrogen molecule from its ground vibrational state $v=0$ to a vibrational state $v=1$ has a value of about $4.45 \cdot 10^{-16} \mathrm{~cm}^{2}$ at $2 \mathrm{eV}$. The swarm analysis of Haddad results in cross sections consistent with the unpublished measurements of Wong et al. of $\mathrm{N}_{2}$ vibrational cross sections (Itikawa et al., 1986). The value of a normalization factor of 0.7 for Schulz's (1976) nitrogen vibrational cross sections is in agreement with the deduction by Aleksandrov et al. (1986) from the analysis of the data on the drift velocity of electrons in a mixture of $\mathrm{Ar}$ and $\mathrm{N}_{2}$.

The vibrational cross sections of $\mathrm{N}_{2}$ can be determined from the results of the differential cross-section (DCS) measurements by extrapolation to $0^{\circ}$ and $180^{\circ}$ followed by integration over the scattering angle. Comparisons of DCS for $\mathrm{N}_{2}(v=0) \rightarrow \mathrm{N}_{2}(v=1)$ scattering from many of these experiments have been carried out by Brennan et al. (1992) and Sun et al. (1995). The discussion given in these papers showed that the positions and shapes of resonant peaks in DCS depend sensitively on both the final vibrational state and the scattering angle. However, the measurements of DCS by Brennan et al. (1992) were performed at only a few energies and used a normalization technique which was revised by Sun et al. (1995). Robertson et al. (1997) used this new technique to calculate vibrational excitation cross sections of $\mathrm{N}_{2}$ and found that their results are in excellent agreement with the cross sections derived from previous swarm analysis and crossed beam studies at energies above 1.5-1.7 eV, but not compatible with the drift velocity data at energies up to about $1 \mathrm{eV}$. The first peak of the $\mathrm{N}_{2}(0) \rightarrow \mathrm{N}_{2}(1)$ cross section has a value of about $4.53 \cdot 10^{-16} \mathrm{~cm}^{2}$ at $1.95 \mathrm{eV}$ (Robertson et al., 
1997) and this value is very close to the value of $4.45 \cdot 10^{-16} \mathrm{~cm}^{2}$ recommended by Haddad (1984).

The primary object of this study is to use the revised $\mathrm{N}_{2}$ cross sections (Schulz, 1976; Haddad, 1984; Robertson et al., 1997) to calculate and to fit to a new analytical expression an energy transfer rate from electrons to $\mathrm{N}_{2}$.

\section{Electron energy transfer rates for vibrational excitation of $\mathrm{N}_{2}$}

The electron cooling rate, $L\left(\mathrm{~N}_{2}^{*}\right)$, arises from the collisions of thermal electrons, $\mathrm{e}_{\mathrm{t}}$, with unexcited $\mathrm{N}_{2}(v=0)$ and vibrationally excited $\mathrm{N}_{2}(v>0)$ molecules. The number densities $\left[\mathrm{N}_{2}(v=0)\right] \gg\left[\mathrm{N}_{2}(v>0)\right]$ during the undisturbed and geomagnetic storm periods at middle and subauroral latitudes (Pavlov and Namgaladze, 1988; Pavlov 1994a, 1996, 1997; Pavlov and Buonsanto, 1997). As a result, the collisions of thermal electrons with unexcited $\mathrm{N}_{2}$ yield the main contribution to the electron cooling rate and the production frequency of the $\mathrm{N}_{2}$ vibrational quanta arising from the collisions of thermal electrons with unexcited and vibrationally excited $\mathrm{N}_{2}$ molecules (Pavlov, 1997). Therefore, in the present paper we restrict our discussion to the transitions from the ground $(v=0)$ and first $(v=1)$ vibrational states of $\mathrm{N}_{2}$ :

$$
\begin{aligned}
& \mathrm{N}_{2}(0)+\mathrm{e}_{\mathrm{t}} \leftrightarrow \mathrm{N}_{2}(v>0)+\mathrm{e}_{\mathrm{t}}, \\
& \mathrm{N}_{2}(1)+\mathrm{e}_{\mathrm{t}} \leftrightarrow \mathrm{N}_{2}(v>1)+\mathrm{e}_{\mathrm{t}},
\end{aligned}
$$

which produce the electron cooling rate

$$
\begin{aligned}
L\left(\mathrm{~N}_{2}^{*}\right)= & N_{e} \sum_{v=1}^{10} Q_{0 v}\left\{\left[\mathrm{~N}_{2}(0)\right]-\left[\mathrm{N}_{2}(v)\right] \exp \left(E_{v} T_{e}^{-1}\right)\right\} \\
& +N_{e} \sum_{v=2}^{9} Q_{1 v}\left\{\left[\mathrm{~N}_{2}(1)\right]-\left[\mathrm{N}_{2}(v)\right]\right. \\
& \left.\times \exp \left(\left(E_{v}-E_{1}\right) T_{e}^{-1}\right)\right\},
\end{aligned}
$$

where

$$
\begin{aligned}
& Q_{0 v}=E_{v}\left\{8 k T_{e}\left(\pi m_{e}\right)^{-1}\right\}^{0.5} \int_{0}^{\infty} \sigma_{0 v}(x) x \exp (-x) \mathrm{d} x \\
& Q_{1 v}=\left(E_{v}-E_{1}\right)\left\{8 k T_{e}\left(\pi m_{e}\right)^{-1}\right\}^{0.5} \int_{0}^{\infty} \sigma_{1 v}(x) x \exp (-x) \mathrm{d} x,
\end{aligned}
$$

$E_{v}=v E_{1}-v(v-1) \Delta E$

$x=E\left(k T_{e}\right)^{-1}, E$ is an energy of electrons, $E_{v}$ is the energy of the $v$-th vibrational level, $\Delta E=20.6 \mathrm{~K}$ is anharmonicity of $\mathrm{N}_{2}$ (Radzig and Smirnov, 1980), $E_{1}=3353 \mathrm{~K}$ (or $0.2889 \mathrm{eV}$ ) is the energy of the first vibrational level of $\mathrm{N}_{2}$ given by Radzig and Smirnov $(1980), \sigma_{0 v}(E)$ are the partial cross sections for excitation of $\mathrm{N}_{2}(0)$ by electrons, $\sigma_{1 v}(E)$ are the partial cross sections for excitation of $\mathrm{N}_{2}(v=1)$ by electrons, $k$ is Boltzmann's coefficient, and $m_{e}$ denotes the mass of electrons.
The results presented in this paper were obtained by use of $\sigma_{01}(E)$ of Engelhard et al. (1964) compiled by Itikawa et al. (1986) for the region of electron energy from 0.3 to $1.7 \mathrm{eV}$ and $\sigma_{01}(E)$ of Robertson et al. (1997) for $E>1.7 \mathrm{eV}$. The cross sections for excitation of $\mathrm{N}_{2}(0)$ to $\mathrm{N}_{2}(2)$ by electrons given by Robertson et al. (1997) were used in our calculations. We used $\sigma_{0 v}(E)$ of the Schulz (1976) for $v=3-10$ with the normalization factor of 0.7 given by Haddad (1984) (when $\sigma_{01}(E)=4.45 \cdot 10^{-16} \mathrm{~cm}^{2}$ at $\left.E=2 \mathrm{eV}\right)$. The partial cross sections for excitation of $\mathrm{N}_{2}(v=1)$ to $\mathrm{N}_{2}(v>1)$ by electrons calculated by Dube and Herzenberg (1979) for $v=2-4$ were adjusted to give the best fit of the calculated $\sigma_{01}(E)$ from Dube and Herzenberg (1979) to the measured $\sigma_{01}(E)$. Chen (1964) calculated $\sigma_{0 v}(E)$ and $\sigma_{1 v}(E)$ for $v=2-9$ and normalized these cross sections to the Schulz (1964) measurements of $\sigma_{0 v}(E)$. In this paper the values of $\sigma_{1 v}(E)$ obtained by Chen (1964) for $v=5-9$ were modified by taking into account the new values of $\sigma_{0 v}(E)$.

The vibrational cross sections used take into account energetically allowed transitions between rotational levels of different vibrational states of $\mathrm{N}_{2}$. The theoretical cross sections $\sigma_{0 v}(E)$ and $\sigma_{1 v}(E)$ equal the sum of rotational-vibrational cross sections for all energetically allowed transitions $0 p \rightarrow v q$ for $\sigma_{0 v}(E)$ and $1 p \rightarrow v q$ for $\sigma_{1 v}(E)$, where $p$ is a number of a $p$-th rotational level and $q$ is a number of a $q$-th rotational level (Robertson et al., 1997). The energy resolution is not sufficient to resolve rotational structure and the discussed measurements of $\sigma_{0 v}(E)$ yield rotationally averaged vibrational excitation cross sections (Sun et al., 1995).

The role of transitions between rotational levels of $\mathrm{N}_{2}(v=0)$ in the ionosphere was studied by Stubbe (1971) and Stubbe and Varnum (1972). Using the formula of Mentzoni and Row (1963) and rotational excitation cross sections given by Gerjuoy and Stein (1955) the rate of electron cooling through rotational excitation was approximated by analytical formula (Stubbe, 1971; Stubbe and Varnum, 1972) which is used in models of the ionosphere. Taking into account the comparison of theoretical and experimental cross sections given by Robertson et al. (1997), this rate of electron cooling must be multiplied by a factor of 1.255 (see Appendix).

Equation (3) includes both excitation and deexcitation, which are related through the principle of detailed balancing. To calculate $\left[\mathrm{N}_{2}(v)\right]$ it is necessary to solve the vibrational quanta continuity equation [a Boltzmann distribution of $\left[\mathrm{N}_{2}(v)\right]$ or the full continuity equations for densities of $\mathrm{N}_{2}(v)$ [a non-Boltzmann distribution of $\mathrm{N}_{2}(v)$ ].

The system of full continuity equations is described by Pavlov (1988, 1994) and Pavlov and Namgaladze (1988) using the harmonic and anharmonic oscillator energy level approximations, the vibrational-vibrational and vibrational-translational energy exchange of $\mathrm{N}_{2}(v)$, the diffusion of $\mathrm{N}_{2}(v)$ in the mixture of $\mathrm{N}_{2}(0), \mathrm{O}_{2}$ and $\mathrm{O}$, and the production rates of $\mathrm{N}_{2}(v)$. The values of the production rates, $q_{v}$, of $\mathrm{N}_{2}(v)$ due to the thermal electron excitation of $\mathrm{N}_{2}(0)$ and $\mathrm{N}_{2}(1)$ and the 
deexcitation of $\mathrm{N}_{2}(v)$ in the reactions given by Eqs. (1) and (2) can be calculated as

$$
\begin{aligned}
q_{1}=N_{e} Q_{01} & E_{1}^{-1}\left\{\left[\mathrm{~N}_{2}(0)\right]-\left[\mathrm{N}_{2}(1)\right] \exp \left(E_{1} T_{e}^{-1}\right)\right\} \\
q_{v}(v>1)= & N_{e} Q_{0 v} E_{v}^{-1}\left\{\left[\mathrm{~N}_{2}(0)\right]-\left[\mathrm{N}_{2}(v)\right] \exp \left(E_{v} T_{e}^{-1}\right)\right\} \\
& +N_{e} Q_{1 v}\left(E_{v}-E_{1}\right)^{-1} \times\left\{\left[\mathrm{N}_{2}(0)\right]\right. \\
& \left.-\left[\mathrm{N}_{2}(v)\right] \exp \left(\left(E_{v}-E_{1}\right) T_{e}^{-1}\right)\right\}
\end{aligned}
$$

where $Q_{0 v}$ and $Q_{1 v}$ are the same as in Eqs. (4) and (5).

In earlier studies, Newton et al. (1974) and Pavlov (1989) found that deviation from a Boltzmann distribution of $\mathrm{N}_{2}(v)$ was large in the SAR-arc region at the vibrational energy levels $v>2$, and this deviation significantly affected the loss rate of the $\mathrm{O}^{+}\left({ }^{4} \mathrm{~S}\right)$ ions at F2-region altitudes. Pavlov and Namgaladze (1988) calculated a vibrational distribution of $\mathrm{N}_{2}(v)$ and found that the difference between the Boltzmann and nonBoltzmann distribution of $\mathrm{N}_{2}(v)$ does not give any effect on the electron density and temperature at solar minimum. During geomagnetic storms at solar maximum the calculated distribution is highly non-Boltzmann at the vibrational levels $j>2$ and the Boltzmann distribution assumption results in the increase of $10-30 \%$ in the calculated F2 peak electron density (Pavlov and Buonsanto, 1997).

The main contribution to $L\left(\mathrm{~N}_{2}^{*}\right)$ arising from the deexcitation of $\mathrm{N}_{2}(v)$ is brought by the deexcitation of $\mathrm{N}_{2}(1)$ and $\mathrm{N}_{2}(2)$. As a result, it is possible to use a Boltzmann distribution of $\mathrm{N}_{2}(v)$ in calculations of $L\left(\mathrm{~N}_{2}^{*}\right)$ :

$\left[\mathrm{N}_{2}(v)\right]=\left[\mathrm{N}_{2}(0)\right]\left\{\alpha(1+\alpha)^{-1}\right\}^{v}=\left[\mathrm{N}_{2}(0)\right] \exp \left(-v T_{\mathrm{vib}}\right)$,

where the vibrational quanta

$\alpha=\sum_{v=1}^{\infty} v\left[\mathrm{~N}_{2}(v)\right] /\left[\mathrm{N}_{2}\right],\left[\mathrm{N}_{2}\right]=\sum_{v=0}^{\infty}\left[\mathrm{N}_{2}(v)\right]$,

and the vibrational temperature $T_{\mathrm{vib}}=-E_{1} / \ln \left[\alpha(1+\alpha)^{-1}\right]$.

In this case, from Eq. (9) it follows that the sum of the vibrationally unexcited and excited molecular nitrogen concentrations (Pavlov and Namgaladze, 1988) is

$\left[\mathrm{N}_{2}\right]=\left[\mathrm{N}_{2}(0)\right](1+\alpha)$.

There is no possibility to differentiate the vibrationally unexcited $\mathrm{N}_{2}$ from the vibrationally excited $\mathrm{N}_{2}$ during the mass-spectrometer measurements of the neutral species in the atmosphere, and therefore it is necessary to point out that the MSIS-86 model (Hedin, 1987), which is usually used in calculations of $L\left(\mathrm{~N}_{2}^{*}\right)$, calculates the value of $\left[\mathrm{N}_{2}\right]$. The difference between $\left[\mathrm{N}_{2}\right]$ and $\left[\mathrm{N}_{2}(0)\right]$ is not negligible for $T_{\text {vib }}>1100-1300 \mathrm{~K}$. For example, $\left[\mathrm{N}_{2}\right] /\left[\mathrm{N}_{2}(0)\right]=1.12$ for $T_{\mathrm{vib}}=1500 \mathrm{~K}$. The difference between $\left[\mathrm{N}_{2}\right]$ and $\left[\mathrm{N}_{2}(0)\right]$ is important in subauroral red arc regions and at solar maximum in the mid-latitude atmosphere.
For the Boltzmann distribution given by Eq. (9)

$$
\begin{aligned}
L\left(\mathrm{~N}_{2}^{*}\right)= & N_{e}\left[\mathrm{~N}_{2}\right]\left\{1-\exp \left(-E_{1} / T_{\mathrm{vib}}\right)\right\} \\
& \times \sum_{v=1}^{10} Q_{0 v}\left\{1-\exp \left[v E_{1}\left(T_{e}^{-1}-T_{\mathrm{vib}}^{-1}\right)\right]\right\} \\
& +N_{e}\left[\mathrm{~N}_{2}\right]\left\{1-\exp \left(-E_{1} / T_{\mathrm{vib}}\right)\right\} \exp \left(-E_{1} / T_{\mathrm{vib}}\right) \\
& \times \sum_{v=2}^{9} Q_{1 v}\left\{1-\exp \left[(v-1) E_{1}\left(T_{e}^{-1}-T_{\mathrm{vib}}^{-1}\right)\right]\right\},
\end{aligned}
$$

To calculate $\alpha$ or $T_{\text {vib }}$ it is necessary to solve the vibrational quanta continuity equation (Pavlov and Namgaladze, 1988; Pavlov, 1988, 1994a). The vibrational-translational energy exchange of $\mathrm{N}_{2}(v)$, the diffusion of $\mathrm{N}_{2}(v)$ in the mixture of $\mathrm{N}_{2}(0), \mathrm{O}_{2}$, and $\mathrm{O}$, and the production frequencies of $\alpha$ are taken into account in the vibrational quanta continuity equation. The values of the production frequency, $W$, of the vibrational quanta due to the thermal electron excitation of $\mathrm{N}_{2}(0)$ and $\mathrm{N}_{2}(1)$ and the deexcitation of $\mathrm{N}_{2}(v)$ can be calculated as

$W=L\left(\mathrm{~N}_{2}^{*}\right)\left\{E_{1}\left[\mathrm{~N}_{2}\right]\right\}^{-1}$.

Pavlov and Buonsanto (1996) studied the effects of vibrationally excited $\mathrm{N}_{2}$ using two approaches: (1) obtaining a full solution of the vibrational quanta continuity equation, and (2) calculating an analytical solution of the steady-state vibrational quanta continuity equation (Pavlov, 1994a) using a simple FORTRAN subroutine. It is shown that during both undisturbed periods and magnetic storms the full solution and the analytical approach give very similar results for the calculated F2 peak electron density and peak height of the F2-layer derived from the time-dependent onedimensional mathematical model of the Earth ionosphere and plasmasphere, so the analytical approach can be recommended for use in ionospheric models during both quiet and disturbed conditions. A FORTRAN subroutine which carries out this steady-state analytical calculation of the vibrational temperature and takes into account the results of this paper is available from the author by e-mail.

Finally, the most simple approach in calculations of $T_{\text {vib }}$ is

$T_{\mathrm{vib}}=T_{n}$,

where $T_{n}$ is a neutral temperature.

The production frequency of $\mathrm{N}_{2}(v)$ arises from the collision of $\mathrm{N}_{2}$ with thermal electrons and $\mathrm{O}\left({ }^{1} \mathrm{D}\right)$ and $\mathrm{N}$ with NO. However, at F-region altitudes a marked difference between $T_{\mathrm{vib}}$ and $T_{n}$ is found only for $T_{e}>1800-2000 \mathrm{~K}$ due to the collisions of $\mathrm{N}_{2}$ with thermal electrons (Pavlov, 1988, 1994a; Pavlov and Namgaladze, 1988). There is absolutely no reason to assert that the condition given by Eq. (13) gives good approximation of $T_{\mathrm{vib}}$ during all geomagnetic conditions, but sometimes (during periods with 
$T_{e}<1800-2000 \mathrm{~K}$ at the F-region altitudes) the Eq.

(13) may be valid with some small errors.

Figures 1 and 2 show $Q_{0 v}$ and $Q_{1 v}$ as functions of $T_{e}$ and $v$. Curves $1-10$ of Fig. 1 are the calculated $Q_{0 v}$ for $v=1-10$ of $\mathrm{N}_{2}(v)$, and curves $2-9$ of Fig. 2 are the calculated $Q_{1 v}$ for $v=2-9$ of $\mathrm{N}_{2}(v)$. The values of $\sum_{v=1}^{10} Q_{0 v}$ and $\sum_{v=2}^{9} Q_{1 v}$ are shown in Figs. 1 and 2 as dashed lines.

Stubbe and Varnum (1972) found the analytical expression for $L\left(\mathrm{~N}_{2}^{*}\right)$ based on the $\mathrm{N}_{2}$ vibrational cross sections of Schulz (1964) and Engelhard et al. (1964) as

$L_{\mathrm{SV}}\left(\mathrm{N}_{2}^{*}\right)=\left[\mathrm{N}_{2}\right] N_{e}\left\{U\left(T_{e}\right)-H\left(T_{e}, T_{n}\right)\right\}$,

where

$$
\begin{aligned}
& U\left(T_{e}\right)=2.99 \cdot 10^{-12} \exp \left\{f\left(5 \cdot 10^{-4}-T_{e}^{-1}\right)\right\}, \\
& H\left(T_{e}, T_{n}\right)=U\left(T_{e}\right) \exp \left\{-g\left(T_{n}^{-1}-T_{e}^{-1}\right)\right\}, \\
& f=1.06 \cdot 10^{4}+7.51 \cdot 10^{3} \tanh \left[10^{-2}\left(T_{e}-1800\right)\right],
\end{aligned}
$$

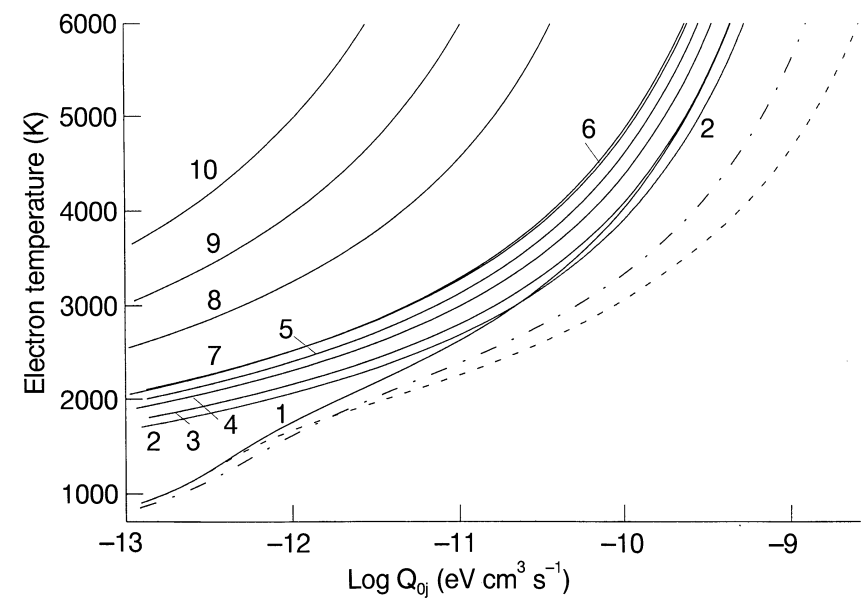

Fig. 1. The calculated $Q_{0 v}$ for the $\mathrm{N}_{2}$ vibrational levels $v=1-10$ (solid curves 1-10). The dashed line is $\sum_{v=1}^{10} Q_{0 v}$ from the calculation of this paper, and the dashed-dotted line is $U\left(T_{e}\right)$ given by Eq. (12) of Stubbe and Varnum (1972)

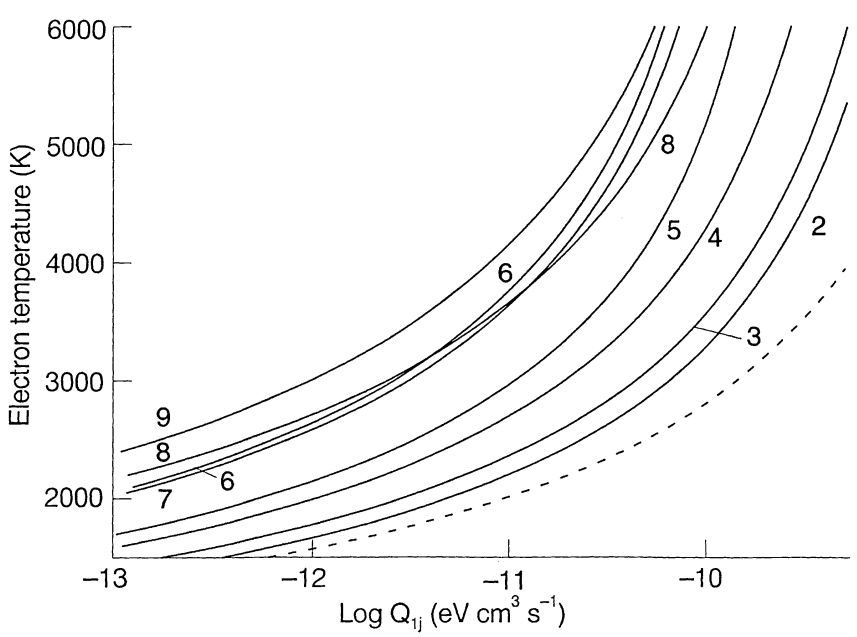

Fig. 2. The calculated $Q_{1 v}$ for the $\mathrm{N}_{2}$ vibrational levels $v=2-9$ (curves 2-9). The dashed line is the calculated value of $\sum_{v=2}^{9} Q_{1 v}$

$$
\begin{aligned}
g= & 3300+1.233\left(T_{e}-1000\right)-2.056 \cdot 10^{-4} \\
& \times\left(T_{e}-1000\right)\left(T_{e}-4000\right),
\end{aligned}
$$

the units of $U\left(T_{e}\right)$ and $H\left(T_{e}, T_{n}\right)$ are $\mathrm{eV} \mathrm{cm}^{3} \mathrm{~s}^{-1}$.

Our results [Eqs. (3)-(6), (11)] for the cooling rate due to $\mathrm{N}_{2}(v)$ can be compared with those of Stubbe and Varnum (1972). From Eqs. (14)-(18) it follows that the analytical expression for $L\left(\mathrm{~N}_{2}^{*}\right)$ was found by Stubbe and Varnum (1972) only for $T_{\text {vib }}=T_{n}$ and without consideration of the difference between $\left[\mathrm{N}_{2}\right]$ and $\left[\mathrm{N}_{2}(0)\right]$. The value of $L_{\mathrm{SV}}\left(\mathrm{N}_{2}^{*}\right)$ includes both excitation $\left(U\left(T_{e}\right)\right)$ and deexcitation $\left(H\left(T_{e}, T_{n}\right)\right)$ of $\mathrm{N}_{2}(v)$ and it is necessary to compare $U\left(T_{e}\right)$ with $\sum_{v=1}^{10} Q_{0 v}$.

The calculated values of $U\left(T_{e}\right)$ and $\sum_{v=1}^{10} Q_{0 v}$ are shown in Fig. 1 as dashed-dotted and dashed lines. The ratio $R=U\left(T_{e}\right) / \sum_{v=1}^{10} Q_{0 v}$ increases with the decrease of $T_{e}$. The value of $R=1.4-1.0$ within the electron temperature range $300-1800 \mathrm{~K}$ and $R=1.0-0.6$ for $1800 \leq T_{e} \leq 3000 \mathrm{~K}$.

The convenient measure of the importance of the reactions in Eqs. (1) and (2) for the electron cooling rate and the production frequency $W$ of the vibrational quanta is the ratio $R=\sum_{v=2}^{9} Q_{1 v} / \sum_{v=1}^{10} Q_{0 v}$. This ratio is 0.14 for $T_{e}=1200 \mathrm{~K}$ and 1.101 for $T_{e}=1500 \mathrm{~K}$. The value of $R$ increases with the increase of $T_{e}$ up to $R=2.59$ for $T_{e}=2000 \mathrm{~K}$, and after that this ratio decreases with the increase of $T_{e}$, reaching $R=1.0$ for $T_{e}=4500 \mathrm{~K}$. The part of cooling rate due to the reaction in Eq. (2) is proportional to $\exp \left(-E_{1} / T_{\text {vib }}\right)$. Therefore, the processes of Eq. (2) can be important for the calculation of $L\left(\mathrm{~N}_{2}^{*}\right)$ and $W$ [in comparison with Eq. (1)] only for very high

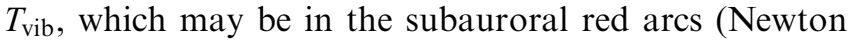
et al., 1974; Pavlov, 1989, 1997).

If the number of vibrational level increases then $Q_{0 v}$ decreases for most values of $T_{e}$. The relative contribution to $L\left(\mathrm{~N}_{2}^{*}\right)$ and $W$ of the vibrational levels $v=8-10$ can be evaluated from the ratio $R=\sum_{v=8}^{10} Q_{0 v} /$ $\sum_{v=1}^{10} Q_{0 v}$. We have $R<0.01$ within the electron temperature range $300-3600 \mathrm{~K}$ and $R<0.02$ for $T_{e} \leq 6000 \mathrm{~K}$. Therefore, the vibrational levels $v=1-7$ are enough to calculate $L\left(\mathrm{~N}_{2}^{*}\right)$ and $W$.

\section{Analytical expressions}

The electron cooling rate, the production rates of $\mathrm{N}_{2}(v)$, and the production frequency of the $\mathrm{N}_{2}$ vibrational quanta arising from the collisions of electrons with unexcited $\mathrm{N}_{2}(0)$ and vibrationally excited $\mathrm{N}_{2}(v>0)$ molecules depend on $Q_{0 v}$ and $Q_{1 v}$ which are functions of the electron temperature. We now give analytical expressions for $Q_{0 v}$ and $Q_{1 v}$ available to the researcher for quick reference and accurate computer modeling with a minimum of calculations:

$$
\begin{aligned}
\log Q_{0 v}(\mathrm{an})= & A_{0 v}+B_{0 v} T_{e}+C_{0 v} T_{e}^{2} \\
& +D_{0 v} T_{e}^{3}+F_{0 v} T_{e}^{4}-16, \\
\log Q_{1 v}(\mathrm{an})= & A_{1 v}+B_{1 v} T_{e}+C_{1 v} T_{e}^{2} \\
& +D_{1 v} T_{e}^{3}+F_{1 v} T_{e}^{4}-16,
\end{aligned}
$$


Table 1. Coefficients for calculations of $Q_{0 v}$ for $1500 \leq T_{e} \leq 6000 \mathrm{~K}$

\begin{tabular}{rrlrrrr}
\hline$v$ & \multicolumn{1}{l}{$A_{0 v}$} & $B_{0 v}, \mathrm{~K}^{-1}$ & \multicolumn{1}{c}{$C_{0 v}, \mathrm{~K}^{-2}$} & \multicolumn{1}{c}{$D_{0 v}, \mathrm{~K}^{-3}$} & \multicolumn{1}{c}{$F_{0 v}, \mathrm{~K}^{-4}$} & $\delta_{0 v}$ \\
\hline 1 & 2.025 & $8.782 \cdot 10^{-4}$ & $2.954 \cdot 10^{-7}$ & $-9.562 \cdot 10^{-11}$ & $7.252 \cdot 10^{-15}$ & 0.06 \\
2 & -7.066 & $1.001 \cdot 10^{-2}$ & $-3.066 \cdot 10^{-6}$ & $4.436 \cdot 10^{-10}$ & $-2.449 \cdot 10^{-14}$ & 0.08 \\
3 & -8.211 & $1.092 \cdot 10^{-2}$ & $-3.369 \cdot 10^{-6}$ & $4.891 \cdot 10^{-10}$ & $-2.706 \cdot 10^{-14}$ & 0.10 \\
4 & -9.713 & $1.204 \cdot 10^{-2}$ & $-3.732 \cdot 10^{-6}$ & $5.431 \cdot 10^{-10}$ & $-3.008 \cdot 10^{-14}$ & 0.10 \\
5 & -10.353 & $1.243 \cdot 10^{-2}$ & $-3.850 \cdot 10^{-6}$ & $5.600 \cdot 10^{-10}$ & $-3.100 \cdot 10^{-14}$ & 0.15 \\
6 & -10.819 & $1.244 \cdot 10^{-2}$ & $-3.771 \cdot 10^{-6}$ & $5.385 \cdot 10^{-10}$ & $-2.936 \cdot 10^{-14}$ & 0.15 \\
7 & -10.183 & $1.185 \cdot 10^{-2}$ & $-3.570 \cdot 10^{-6}$ & $5.086 \cdot 10^{-10}$ & $-2.769 \cdot 10^{-14}$ & 0.15 \\
8 & -12.698 & $1.309 \cdot 10^{-2}$ & $-3.952 \cdot 10^{-6}$ & $5.636 \cdot 10^{-10}$ & $-3.071 \cdot 10^{-14}$ & 0.15 \\
9 & -14.710 & $1.409 \cdot 10^{-2}$ & $-4.249 \cdot 10^{-6}$ & $6.058 \cdot 10^{-10}$ & $-3.300 \cdot 10^{-14}$ & 0.15 \\
10 & -17.538 & $1.600 \cdot 10^{-2}$ & $-4.916 \cdot 10^{-6}$ & $7.128 \cdot 10^{-10}$ & $-3.941 \cdot 10^{-14}$ & \\
\hline
\end{tabular}

Table 2. Coefficients for calculations of $Q_{0 v}$ for $300 \leq T_{e} \leq 1500 \mathrm{~K}$

\begin{tabular}{lllllll}
\hline$v$ & $A_{0 v}$ & $B_{0 v}, \mathrm{~K}^{-1}$ & $C_{0 v}, \mathrm{~K}^{-2}$ & $D_{0 v}, \mathrm{~K}^{-3}$ & $F_{0 v}, \mathrm{~K}^{-4}$ & $\delta_{0 v}$ \\
\hline 1 & -6.462 & $3.151 \cdot 10^{-2}$ & $-4.075 \cdot 10^{-5}$ & $2.439 \cdot 10^{-8}$ & $-5.479 \cdot 10^{-12}$ & 0.14 \\
\hline
\end{tabular}

Table 3. Coefficients for calculations of $Q_{1 v}$ for $1500 \leq T_{e} \leq 6000 \mathrm{~K}$

\begin{tabular}{lllllll}
\hline$v$ & $A_{1 v}$ & $B_{1 v}, \mathrm{~K}^{-1}$ & $C_{1 v}, \mathrm{~K}^{-2}$ & $D_{1 v}, \mathrm{~K}^{-3}$ & $F_{1 v}, \mathrm{~K}^{-4}$ & $\delta_{1 v}$ \\
\hline 2 & -3.413 & $7.326 \cdot 10^{-3}$ & $-2.200 \cdot 10^{-6}$ & $3.128 \cdot 10^{-10}$ & $-1.702 \cdot 10^{-14}$ & 0.11 \\
3 & -4.160 & $7.803 \cdot 10^{-3}$ & $-2.352 \cdot 10^{-6}$ & $3.352 \cdot 10^{-10}$ & $-1.828 \cdot 10^{-14}$ & 0.11 \\
4 & -5.193 & $8.360 \cdot 10^{-3}$ & $-2.526 \cdot 10^{-6}$ & $3.606 \cdot 10^{-10}$ & $-1.968 \cdot 10^{-14}$ & 0.12 \\
5 & -5.939 & $8.807 \cdot 10^{-3}$ & $-2.669 \cdot 10^{-6}$ & $3.806 \cdot 10^{-10}$ & $-2.073 \cdot 10^{-14}$ & 0.08 \\
6 & -8.261 & $1.010 \cdot 10^{-2}$ & $-3.039 \cdot 10^{-6}$ & $4.318 \cdot 10^{-10}$ & $-2.347 \cdot 10^{-14}$ & 0.10 \\
7 & -8.185 & $1.010 \cdot 10^{-2}$ & $-3.039 \cdot 10^{-6}$ & $4.318 \cdot 10^{-10}$ & $-2.347 \cdot 10^{-14}$ & 0.12 \\
8 & -10.823 & $1.199 \cdot 10^{-2}$ & $-3.620 \cdot 10^{-6}$ & $5.159 \cdot 10^{-10}$ & $-2.810 \cdot 10^{-14}$ & 0.09 \\
9 & -11.273 & $1.283 \cdot 10^{-2}$ & $-3.879 \cdot 10^{-6}$ & $5.534 \cdot 10^{-10}$ & $-3.016 \cdot 10^{-14}$ & 0.09 \\
\hline
\end{tabular}

where the constants $A_{0 v}, B_{0 v}, C_{0 v}, D_{0 v}, F_{1 v}, A_{1 v}, B_{1 v}, C_{1 v}$, $D_{1 v}$, and $F_{1 v}$ are given in Tables $1-3$, the units of $Q_{0 v}($ an $)$ and $Q_{1 v}($ an $)$ are $\mathrm{eV} \mathrm{cm}^{3} \mathrm{~s}^{-1}$, the units of $T_{e}$ are K.

To establish how good or bad the analytical Eqs. (11) and (12) can be, it is necessary to define quantitative measure of maximum error for given range of $T_{e}$ as

$\delta_{0 v}=\max \mid 1-Q_{0 v}($ an $) / Q_{0 v} \mid$,

$\delta_{1 v}=\max \left|1-Q_{1 v}(\mathrm{an}) / Q_{1 v}\right|$.

The calculated values of $\delta_{0 v}$ and $\delta_{1 v}$ are presented in Tables 1-3.

The ratio $Q_{01} / \sum_{v=1}^{10} Q_{0 v}>0.94$ for $T_{e}<1500 \mathrm{~K}$. The vibrational temperature of $\mathrm{N}_{2}$ is close to the neutral temperature for $T_{e}<1500 \mathrm{~K}$ (Pavlov, 1988, 1994a; Pavlov and Namgaladze, 1988). Therefore the processes of Eqs. (1) and (2) with $v>1$ are unimportant for the calculation of $L\left(\mathrm{~N}_{2}^{*}\right)$ and $W$ for $T_{e}<1500 \mathrm{~K}$.

\section{Conclusions}

The electron energy transfer rates for vibrational excitation of $\mathrm{N}_{2}$ have been calculated and fitted to analytical expressions by the use of the revised vibrationally excited $\mathrm{N}_{2}$ cross sections (Schulz, 1976; Haddad, 1984). Our results for the cooling rate due to $\mathrm{N}_{2}(v)$ are smaller than those of Stubbe and Varnum (1972) in the $\mathrm{E}$ region of the ionosphere and at F-region altitudes for $T_{e}<1800 \mathrm{~K}$. The new cooling rate is larger than the widely used Stubbe and Varnum (1972) cooling rate for $T_{e}>1800 \mathrm{~K}$. We found that the processes given by Eq. (1) give the main contribution to the values of the electron cooling rate and the production frequency of the $\mathrm{N}_{2}$ vibrational quanta arising from the collisions of electrons with unexcited $\mathrm{N}_{2}(0)$ and vibrationally excited $\mathrm{N}_{2}$ (1) molecules. The processes of Eq. (2) can be important for the calculation of $L\left(\mathrm{~N}_{2}^{*}\right)$ and $W$ [in comparison with Eq. (1)] only for very high $T_{\text {vib }}$, which may be in the subauroral red arcs. We have also shown that the relative contribution of the vibrational levels $v=8-10$ to the electron cooling rate and the production frequency of the $\mathrm{N}_{2}$ vibrational quanta is less $1 \%$ for $T_{e} \leq 3600 \mathrm{~K}$ and $2 \%$ for $T_{e} \leq 6000 \mathrm{~K}$. Therefore, the vibrational levels $v=1-7$ are enough to calculate $L\left(\mathbf{N}_{2}^{*}\right)$ and $\mathrm{W}$.

The electron cooling rate due to rotational excitation of $\mathrm{N}_{2}(v=0)$ was found by Stubbe (1971) and Stubbe and Varnum (1972). It is shown that this rate of electron energy loss associated with rotational transitions in $\mathrm{N}_{2}$ must be multiplied by a factor or 1.255.

Acknowledgements. The research described in this publication was supported by grant 96-05-64031 from the Russian Foundation of the Fundamental Researches. I would like to thank anonymous referees and Prof. M. A. Morrison for detailed and constructive comments which have helped improve the paper.

Topical Editor D. Alcaydé thanks M. Morrison and another referee for their help in evaluating this paper. 


\section{Appendix}

\section{The electron cooling rate by means of rotational excitation of $\mathrm{N}_{2}$}

The general expression for the rate of electron energy loss associated with rotational transitions in $\mathrm{N}_{2}(v=0)$ is given by Eq. (1) of Stubbe and Varnum (1972). Using the quadrupole Born approximation formula for rotational excitation cross sections given by Gerjuoy and Stein (1955), and making use of the fact that the rotational states are so close together that the summation can be changed into an integration (Mentzoni and Row, 1963), the electron cooling rate by means of rotational excitation of $\mathrm{N}_{2}(v=0)$ can be calculated as

$L_{\mathrm{rot}}\left(\mathrm{N}_{2}(v=0)\right)=C\left[\mathrm{~N}_{2}(v=0)\right] N_{e}\left(T_{e}-T_{n}\right) T_{e}^{-0.5}$,

where $C=\left(64 \pi B a_{0}^{2} Q^{2} 2^{0.5}\right) /\left(15 \pi^{0.5} m_{e}^{0.5}\right), B$ is the rotational constant for the ground vibrational state of $\mathrm{N}_{2}, Q$ is the quadrupole moment averaged over the ground vibrational state $\mathrm{N}_{2}, a_{0}$ is the Bohr radius.

Mentzoni and Row (1963) used the values of $B=2.49 \cdot 10^{-4} \mathrm{eV}$ and $Q=-0.98 e a_{0}^{2}$, where $e$ is the electronic charge. As a result, Stubbe (1971) and Stubbe and Varnum (1972) found $C=2.8 \cdot 10^{-14} \mathrm{eV} \mathrm{cm}^{3} \mathrm{~s}^{-1}$ $\mathrm{K}^{-0.5}$ where the units of $\left[\mathrm{N}_{2}\right]$ and $N_{e}$ are $\mathrm{cm}^{-3}$, the units of $L_{\mathrm{rot}}\left(\mathrm{N}_{2}(v=0)\right)$ are $\mathrm{eV} \mathrm{cm}^{-3} \mathrm{~s}^{-1}$, and the units of $T_{e}$ are $\mathrm{K}$.

Robertson et al. (1997) treated the quadrupole moment $Q$ as an adjustable parameter and obtained the best fit of the quadrupole Born approximation for rotational excitation cross sections to the experimental cross sections with a value of $Q=-1.1 e a_{0}^{2}$. This value of $Q$ and more accurate value of $B=2.4805 \cdot 10^{-4} \mathrm{eV}$ (Robertson et al., 1997) give $C=3.5 \cdot 10^{-14} \mathrm{eV} \mathrm{cm}^{3} \mathrm{~s}^{-1}$ $\mathrm{K}^{-0.5}$

The total rate of electron energy loss associated with rotational transitions within each vibrational level of $\mathrm{N}_{2}$ is $L_{\mathrm{rot}}\left(\mathrm{N}_{2}\right)=\sum_{v=0}^{\infty} L_{\mathrm{rot}}\left(\mathrm{N}_{2}(v)\right)$. To simplify the problem, Stubbe (1971) and Stubbe and Varnum (1972) used

$L_{\mathrm{rot}}\left(\mathrm{N}_{2}\right)=C\left[\mathrm{~N}_{2}\right] N_{e}\left(T_{e}-T_{n}\right) T_{e}^{-0.5}$,

meaning that they did not distinguish between rotational excitation cross sections of $\mathrm{N}_{2}(v=0)$ and $\mathrm{N}_{2}(v>0)$ (as far as the author knows, rotational excitation cross sections of $\mathrm{N}_{2}(v>0)$ are not measured). The number densities give $\left[\mathrm{N}_{2}(v=0)\right] \gg\left[\mathrm{N}_{2}(v>0)\right]$ during the undisturbed and geomagnetic storm periods at middle and subauroral latitudes (Pavlov and Namgaladze, 1988; Pavlov, 1994a, 1996, 1997; Pavlov and Buonsanto, 1997). As a result, the collisions of thermal electrons with vibrationally unexcited $\mathrm{N}_{2}$ yield the main contribution for the rate of electron energy loss associated with rotational transitions within $\mathrm{N}_{2}(v=0)$ and $\mathrm{N}_{2}(v>0)$, and the possible error or Eq. (A2) is expected to be negligible.

\section{References}

Aleksandrov, N. L., I. V. Kochetov, and A. P. Naportovich, Normalization of cross sections for vibrational excitation of $\mathrm{N}_{2}$ by electron impact (in Russian), Chem. High Energies, 20, 291-293, 1986.

Brennan, M. J., D. T. Alle, P. Euripides, S. J. Buckman, and M. J. Brunger, Elastic electron scattering and rovibrational excitation of $\mathrm{N}_{2}$ at low incident energies, J. Phys. B, 25, 2669-2682, 1992.

Chen, J. C. Y., Theory of subexcitation electron scattering by molecules II. Excitation and deexcitation of molecular vibration, J. Chem. Phys., 40, 3513-3520, 1964.

Dube, L., and A. Herzenberg, Absolute cross sections from the "boomerang model" for resonant electron-molecule scattering, Phys. Rev. A, 20, 194-213, 1979.

Engelhardt, A. G., A. V. Phelps, and C. G. Risk, Determination of momentum transfer and inelastic collision cross sections for electrons in nitrogen using transport coefficients, Phys. Rev. A, 135, 1556-1574, 1964.

Gerjuoy, E., and S. Stein, Rotational excitation by slow electrons, Phys. Rev. A, 97, 1671-1679, 1955.

Haddad, G. N., Cross sections for electron scattering in nitrogen, Austr. J. Phys., 37, 484-494, 1984.

Hedin, A. E., MSIS-86 thermospheric model, J. Geophys. Res., 92, 4649-4662, 1987.

Itikawa, Y., M. Hayashi, A. Ichimura, K. Onda, K. Sakimoto, T. Takayanagi, M. Nakamura, H. Nishimura, and T. Takayanagi, Cross sections for collisions of electrons and photons with nitrogen molecules, J. Phys. Chem. Ref. Data, 15, 985-1010, 1986.

Mentzoni, M. H., and R. V. Row, Rotational excitation and electron relaxation in nitrogen, Phys. Rev. A, 130, 2312-2316, 1963.

Newton, G. P., J. C. G. Walker, and P. H. E. Meijer, Vibrationally excited nitrogen in stable auroral red arcs and its effect on ionospheric recombination, J. Geophys. Res., 79, 3807-3818, 1974.

Pavlov, A. V., The role of vibrationally excited nitrogen in the ionosphere, Pure Appl. Geophys., 127, 529-544, 1988.

Pavlov, A. V., About the role of vibrationally excited nitrogen in the subauroral red arc region (in Russian), Geomagn. Aeron., 29, 948-953, 1989.

Pavlov, A. V., The role of vibrationally excited nitrogen in the formation of the mid-latitude ionospheric storms, Ann. Geophysicae, 12, 554-564, 1994a.

Pavlov, A. V., The role of vibrationally excited oxygen and nitrogen in the D and E regions of ionosphere, Ann. Geophysicae, 12, 1085-1090, 1994b.

Pavlov, A. V., Mechanisms of the electron density depletion in the SAR arc region, Ann. Geophysicae, 14, 211-221, 1996.

Pavlov, A. V., Subauroral red arcs as a conjugate phenomenon: comparison of OV1-10 satellite data with numerical calculations, Ann. Geophysicae, 15, 984-998, 1997.

Pavlov, A. V., and M. J. Buonsanto, Using steady-state vibrational temperatures to model effects of $\mathrm{N}_{2}^{*}$ on calculations of electron densities, J. Geophys. Res., 101, 26941-26945, 1996.

Pavlov, A. V., and M. J. Buonsanto, Comparison of model electron densities and temperatures with Millstone Hill observations during undisturbed periods and the geomagnetic storms of March 16-23 and April 6-12, 1990, Ann. Geophysicae, 15, 327344, 1997.

Pavlov, A. V., and A. A. Namgaladze, Vibrationally excited nitrogen in the upper atmosphere. Review paper (in Russian), Geomagn. Aeron., 28, 705-721, 1988.

Radzig, A. A., and B. V. Smirnov, The reference book in atomic and molecular physics (in Russian), Atomizdat, Moscow, 1980.

Robertson, A. G., M. T. Elford, R. W. Crompton, M. A. Morrison, W. Sun, and W. K. Trail, Rotational and vibrational excitation of nitrogen by electron impact, Aust. J. Phys., 50, 441-472, 1997. 
Schulz, G. J., Vibrational excitation of $\mathrm{N}_{2}, \mathrm{CO}$ and $\mathrm{H}_{2}$ by electron impact, Phys. Rev. A, 135, 988-994, 1964.

Schulz, G. J., A review of vibrational excitation of molecules by electron impact at low energies, in Principles of laser plasmas, Ed. G. Berkefi, Interscience, New York, pp. 33-76, 1976.

Stubbe, P., Energy exchange and thermal balance problems, J. Sci. Ind. Res., 30, 379-387, 1971.
Stubbe, P., and W. S. Varnum, Electron energy transfer rates in the ionosphere, Planet. Space Sci., 20, 1121-1126, 1972.

Sun, W., M. A. Morrison, W. A. Issaacs, W. K. Trail, D. T. Alle, R. J. Gulley, M. J. Brennan, and S. J. Stephen, Detailed theoretical and experimental analysis of low-energy electron- $\mathrm{N}_{2}$ scattering, Phys. Rev. A, 52, 1229-1256, 1995. 